Prof. Dr. Michael Linden
Forschungsgruppe Psychosomatische Rehabilitation
Charité Universitätsmedizin Berlin;
Abteilung Verhaltenstherapie und Psychosomatik,
Rehazentrum Seehof, Teltow/Berlin

\title{
Wer gesund ist, erträgt Krankheit leichter
}

- Theoretische Ansätze zur Definition von Gesundheit und daraus abgeleitete Strategien zur Erhaltung, Stärkung und Wiederherstellung von Gesundheit haben eine lange Tradition, die mit der griechischen Antike beginnt und über Aufklärung und Romantische Medizin (z. B. Hufeland) zur Lebensreformbewegung des beginnenden 20. Jahrhunderts mit ihren Überlegungen zur "Hygiene und Psychohygiene" reicht.

Maßnahmen zum Erhalt und zur Förderung der Gesundheit und Vorbeugung von Krankheit sind Aufgaben der alltäglichen Lebensführung. Sie umfassen Bewegung, Ernährung und die psychosoziale Gesundheitsförderung. Diese geht fließend über in Lifestyle- oder Wellnessaktivitäten. Darunter können alle Maßnahmen zur Verwirklichung eines Lebensstils verstanden werden, die das Ziel haben,

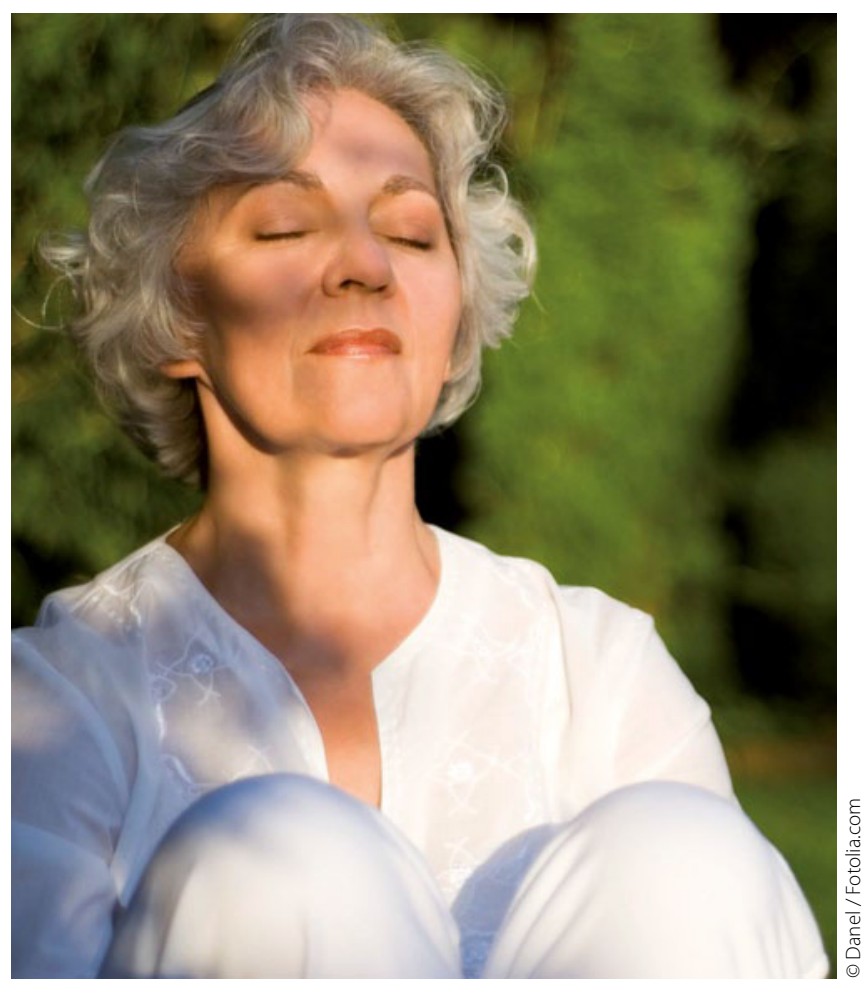

über eine Gesunderhaltung hinaus das eigene Wohlbefinden zu verbessern.

Von Gesundheitsförderung und Lifestyle-Aktivitäten ist schließlich die "Salutotherapie" abzugrenzen. Voraussetzung hierfür ist das Vorliegen einer Krankheit, in deren Folge es zu einer Gesundheitsbeeinträchtigung gekommen ist. Gerade bei langandauernden und chronischen Erkrankungen gilt das „Kuckucksei-Syndrom“. Wegen der Krankheit wird das tägliche Leben zunehmend eingeengt, die Selbstfürsorge lässt nach, Sozialkontakte schwinden und die Lebensqualität leidet zusätzlich. Des Weiteren gilt auch, dass eine Behandlung der Krankheit nicht zugleich auch zu einer Wiederherstellung von Gesundheit führt. Es gilt der Grundsatz, dass Krankheit (z.B. Herzinsuffizienz oder Angststörung) umso besser zu ertragen ist, je gesünder ein Mensch ist, $d$. h. wenn er sich ansonsten in einem guten körperlichen Allgemeinzustand befindet, psychisch ausgeglichen ist, über gute Sozialbeziehungen verfügt oder kulturelle Interessen hat. Eine alleinige krankheitsorientierte Therapie ist in aller Regel nicht hinreichend, sondern muss durch eine gesundheitsorientierte Salutotherapie ergänzt werden. Nur durch Unkraut rupfen erhält man keinen Blumengarten, man muss zusätzlich Blumen pflanzen und hegen.

Salutotherapie geht von einem ganzheitlich biopsychosozialen Verständnis der menschlichen Existenz aus. Sie berücksichtigt Selbstheilungskräfte und Ressourcen der betroffenen Person ebenso wie Bezüge zur sozialen Umgebung im Nahbereich und zum größeren soziokulturellen Kontext. Das Spektrum salutotherapeutischer Ansätze ist breit und reicht von der Förderung eng definierter Fähigkeiten wie Selbstsicherheit oder Körperkraft über den Aufbau von Alltagsaktivitäten bis hin zu sinnorientierten Therapiemaßnahmen. Hierfür gibt es umfangreiche Konzepte, operationalisierbare Interventionen und wissenschaftliche Evidenz. Salutotherapie ist ein unverzichtbarer Behandlungsbaustein in der Rehabilitationsmedizin. Das Konzept der Gesundheitsentwicklung trotz oder wegen unabänderlichen Krankseins verhindert auch Pessimismus in der Behandlung chronischer Krankheiten. Allerdings verdient die Salutotherapie in der Ausbildung von Ärzten, in der täglichen Praxis und auch in der Forschung sicher noch deutlich mehr Aufmerksamkeit. 